\title{
Application and Study on Sentiment-oriented Analysis on Social Semantic Network*
}

\author{
Dan Zhang ${ }^{1}$, Dongsheng Wang ${ }^{2}$, Haiping Huang ${ }^{3,4}$ \\ ${ }^{1}$ College of Telecommunications \& Information Engineering, Nanjing University of Posts and Telecommunications, Nanjing, \\ China \\ ${ }^{2}$ Department of Computer and Information Science Korea University Seoul, South Korea \\ ${ }^{3}$ College of Computer \& Information Science, Nanjing University of Posts and Telecommunications Nanjing, China \\ ${ }^{4}$ Jiangsu Computer Information Processing Technology Key Laboratory, Suzhou University Suzhou, China \\ fionachang92@gmail.com, dswang2011@korea.ac.kr, hhp@njupt.edu.cn
}

\begin{abstract}
With the increasing growth of users and exponential propagation of messages, Social Network Services such as Twitter, Facebook can be used to analyze social trend, people's interests and personal-favor. Recently, the Social Network Analysis based on Semantic Web technology tends to be a hot study. In this paper, we propose a sentiment-oriented method that takes advantage of emotion ontology to reason out Twitter users' basic emotions and retrieve $Y A G O$ ontology to explain associative topics. We implement a prototype system that visualizes the analysis result, which is humanreadable as well as machine-understandable.

Index Terms - Semantic Web technology, Twitter Mining, Social Network Analysis, emotion analysis, Social Semantic Network.
\end{abstract}

\section{Introduction}

The development of Social Network Service (SNS) of web 2.0 has revolutionized our lives. For example, Facebook, YouTube and Google have become part of most people's daily life. Data mining techniques and analysis on SNSs have been studied for years. Meanwhile semantic web technology is a innovative technology, the essence of which is split from Artificial Intelligence (AI). With the Semantic Web technology on its way to develop, the study of applying it on Social Network Analysis is raised, which is commonly called as Social Semantic Network (SSN) such as [1].

We propose a sentiment-oriented analysis method that takes advantage of ontology to reason out Twitter users' emotion into basic words and perform associative topic searching on YAGO ontology. That is, for users' emotions, we use OntoEmotion [2] to reason them into their basic emotion so that it can be understood easily. For the topics, or nouns, associated with the emotion we search them on YAGO ontology to explain what the topics mean.

We implement a prototype system that visualizes the result and demonstrates the relation among emotion, topics and their explanations. It shows a human-friendly as well as machinereadable result, which can benefit the intelligent analysis and further application.

\section{Related Works}

Social Network contains a wealth of data ranging from users to organizations, which is reflecting the real society. Data mining from Social Network has been studies for years. For example, the study of topological characteristics of Twitter in [3] crawled the entire Twitter site and obtained 41.7million user profiles, 1.47 billion social relations. And the study in [4] crawled Facebook data in order to extract a significant sample of users and relations.

Sentiment based analysis on SNS have also attracted many researchers. The level of emotion intensity for communications on social media are estimated in [5]. In [6], Twitter message is identified as positive, negative or neutral ones. Authors in [7] employ large number of influential users' reply tweets to determine audience sentiment. However, people's emotion cannot be explained by singly positive or negative since human's emotion is abundant.

Semantic web technology is largely employed in many applications, for example, a framework in [8] is designed for the semantically-enabled web applications. Web Ontology Language (OWL) is the primary ontology language that enables data machine-understandable. The various language elements of OWL are described in [9] in detail. SPARQL [10] is a query Language for RDF as a formal standard that support semantic search on ontology data.

There are some significant studies on emotion tools and ontology. The Emotion Markup Language (Emotion ML) is developed by The W3C (World Wide Web Consortium). Human Emotion Ontology (HEO) [11] supplies a wide set of properties to describe emotions by category. Verginia supported their emotion ontology which is called the OntoEmotion [2], as illustrated in Fig. 1. This hierarchy in ontology helps us identify whether two emotions are the same, similar or totally distinct. We employ it to reason emotions into their basic emotions so that it can be understood easily. As Fig. 1 shows, basic emotion includes Happiness, Sadness, Fear, Surprise, etc. There are 157 emotion instances supported, and we enrich them into 326 manually.

\footnotetext{
* This subject was sponsored by the National Natural Science Foundation of P.R. China (No. 61003039), Fund of Jiangsu Provincial Key Laborat ory for Computer Information Processing Technology (KJS 1022) and Scientific and Technological Support Project (Industry) of Jiangsu Province (No. BE2012183).
} 


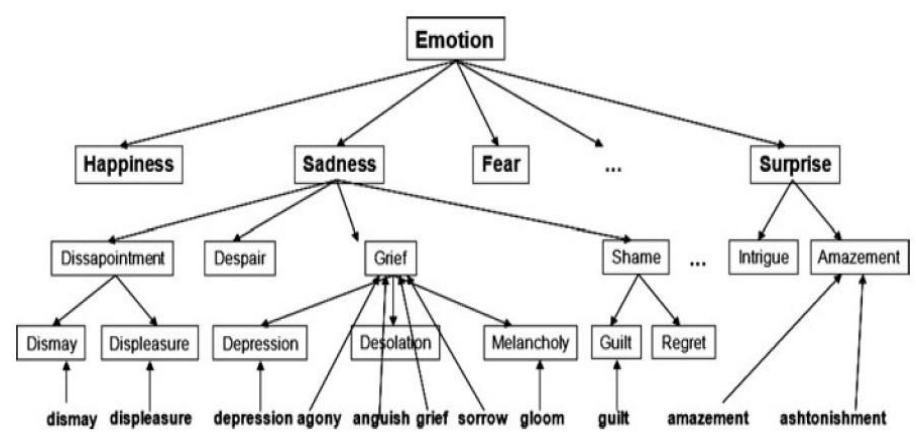

Fig. 1 Fragment of OntoEmotion.

\section{Sentiment-oriented Analysis on SSN}

The overall architecture is proposed as Fig 2 illustrated. From raw data mining to ontology retrieving, it contains Natural Language (NL) processing, RDF Filter, ontology binding and reasoning, and SPARQL search.

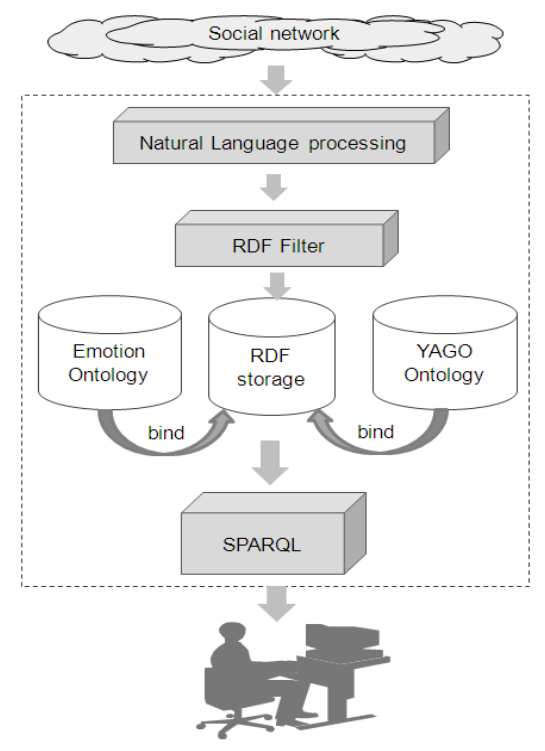

Fig. 2 Overall architecture.

- NL processing: NL processing includes part-of-speech tagging, stop word removing, stemming and etc. This step is aimed to get meaningful keywords and their relationship.

- RDF filter: This step is aimed to transfer keywords and their relationship into RDF format and store them in RDF storage. Jena ${ }^{2}$ framework is employed to implement this module.

- RDF storage: There are many tools that support RDF data storage, and it is officially suggested by Jena framework to employ the Tuple Data Base (TDB) to store RDF data.

- Emotion Ontology: as demonstrated in Fig. 1, we employ this hierarchy ontology to help us reason emotions into their basic emotions (such as happiness, sadness, fear etc.) so that it can be understood easily.
- $Y A G O$ ontology: Topics associated with emotions perform searching on $Y A G O$. In this way, sentiment-oriented topics can be explained in more details in a linked graph.

- SPARQL: Semantic retrieving on ontology data is performed through SPARQL language.

As shown in Fig. 1, the basic emotions are: sadness, happiness, surprise, fear, anger, affection, bravery, disgust and neutral (which is not an emotion, representing the absence of emotion). If an emotion is related to one of the ontology emotions, the word searches its supper class (SubClassOf) till the first layer, namely, basic emotions. And it is replaced by its basic emotion. Then we aggregate the amount for each basic emotion to analyze the frequency of them.

For example, for the tweet "I am so glad that Barack Obama won the campaign again," Natural Language processing gives us such a result: \{( glad, adjective $)$, (Barack_Obama, Noun), (campaign, noun)\}. The relationship between the three words is simply defined as "associative". For the emotion "glad", it is reasoned to its basic emotion, that is, "happiness". For the Barack_Obama, it searches YAGO and gives us terms such as Harvard_Law_School_alumni, African_American_Plotician, etc. Therefore, a tweet message gives us terms linked from emotion to topics and its explanations. We show a practical running result in section 4 .

\section{Performance Study}

We implement a prototype system based on the Twitter application programming interface. Fig. 3 shows the screenshot of the system. We crawl about 50,000 Twitter profiles from Hawaii and show some analysis results. Section 4.1 shows the emotion reasoning that demonstrates the distribution of basic emotions. Section 4.2 shows the linked relations between emotions, topics and associated explanations.

\section{A. Emotion Ontology based reasoning}

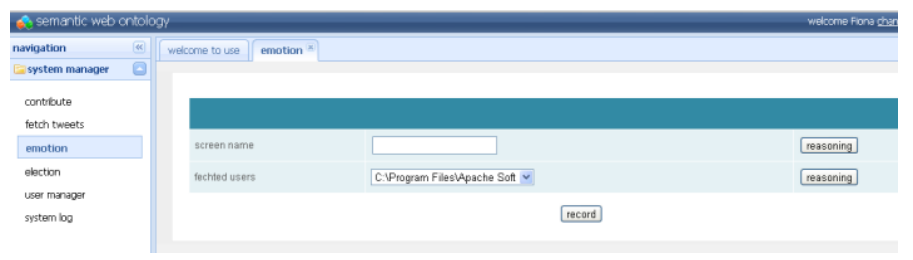

Fig. 3 Screenshot of the application

As discussed in section 3, the system reasons all the emotions that can be mapped into ontoEmotion ontology into their basic emotion. In other words, the emotion will be replaced by its basic emotion if available through the relation of "subClassOf" till the end. Then each of them is aggregated and the distribution of the six basic emotions can be illustrated easily. Fig. 4 shows the basic emotion reasoning result for Hawaii user in June 2011.

\footnotetext{
${ }^{2}$ http://jena.apache.org/
} 


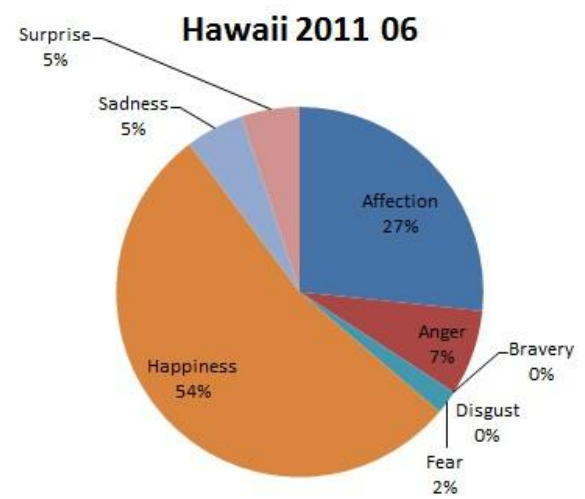

Fig. 4 Screenshot of the emotion analysis for Hawaii user data on 2011.06

\section{B. Associated topics retrieving}

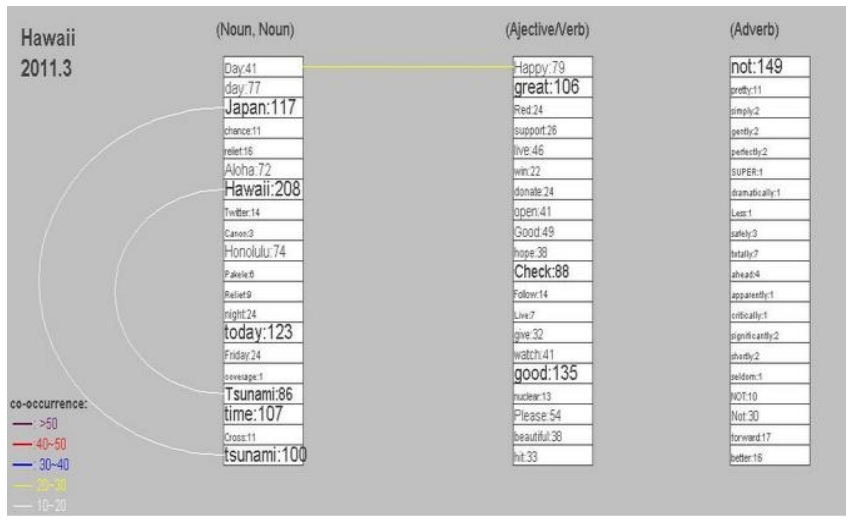

Fig. 5 Screenshot of the emotion and nouns

We found that the emotion in March 2011 is very down, therefore we run the associated topic analysis which shows that the tsunami in Japan affects Hawaii people largely. Fig. 5 shows that the Japan Tsunami becomes a hot issue among Hawaii. The font size of the word means the appearance frequency of the word, and the more times a word appears the bigger it is. Different color of the line indicates different cooccurrence as illustrated in Fig 5. White color line indicate the co-occurence frequency of the two words is between 10 and 20 , yellow line is between 20 and 30 , blue line is between 30 and 40 , etc.

In case that the nouns may not be known by all people, we can perform search of "Japan" on YAGO, and the result explains "Japan" as table I shows. In same way, we search the "tsunami" as table II shows.

TABLE I “Japan" Retrieval of YAGO

\begin{tabular}{|l|l|}
\hline Predicate & object \\
\hline Type & wikicategory_Island_countries \\
\cline { 2 - 3 } (is_a) & wikicategory_OECD_member_economies \\
\cline { 2 - 2 } & wikicategory_Countries_bordering_the_Pacific_Ocean \\
\cline { 2 - 2 } & wikicategory_East_Asian_countries \\
\cline { 2 - 2 } & wikicategory_States_and_territories_established_in_660_BC \\
\cline { 2 - 2 } & wikicategory_Countries_bordering_the_Philippine_Sea \\
\cline { 2 - 2 } & wikicategory_Empires \\
\hline
\end{tabular}

TABLE II “Tsunami” Retrieval of YAGO

\begin{tabular}{|l|l|}
\hline Predicate & object \\
\hline hasGloss & $\begin{array}{l}\text { a cataclysm resulting from a destructive sea wave caused by } \\
\text { an earthquake or volcanic eruption; } \backslash \text { "a colossal tsunami } \\
\text { destroyed the Minoan civilization in minutes।" }\end{array}$ \\
\hline subClassOf & wordnet_calamity_107314838 \\
\hline subClassOf & wordnet_wave_107352190 \\
\hline
\end{tabular}

\section{Conclusions}

We introduced the SNS and Semantic Web, followed by their combination technology, namely, SSN. Based on SSN, we proposed the sentiment-oriented method with an architecture that takes advantage of OWL reasoning and semantic searching on ontology. For emotions, we reason them into their basic ones so that it can be understood easily. For the nouns associated with emotions, we explored their relations and explain them by retrieving $Y A G O$ ontology. In this way, the messages on SSN can be machine-understandable and be analyzed easily.

The prototype system implemented by us shows that the result is human-readable as well as machine-understandable, which can benefit the intelligent analysis and further application in various domains.

\section{References}

[1] P. Mika, "Social networks and the semantic web," IEEE/WIC/ACM International Conference, 2004, pp. 285-291.

[2] V. Francisco, R. Hervás, F. Peinado and P. Gervás, "EmoTales: creating a corpus of folk tales with emotional annotations," Language Resources and Evaluation, vol.46, no. 3, pp. 341-381, 2012.

[3] H. Kwak, C. Lee, H. Park, and S. Moon, "What is twitter, a social network or a news media?" $19^{\text {th }}$ International Conf. on World Wide Web, 2010.

[4] S. A. D. M. C. Pasquale, F. Emilio, F. Giacomo and P. Alessandro, "Crawling Facebook for Social Network Analysis Purposes," ArXiv Eprints. 2011.

[5] G. Paltoglou and M. Thelwall, "Twitter, My Space, Digg: Unsupervised sentiment analysis in social media," ACM Transactions on Intelligent Systems and Technology, vol. 3, 2012.

[6] A. Go, R. Bhayani and L. Huang, "Twitter sentiment classification using distant supervision," Machine Learning, pp. 1-4, 2009.

[7] Y. Bae and H. Lee, "Sentiment analysis of twitter audiences: measuring the positive or negative influence of popular twitterers," Journal of the American Society for Information Science and Technology. Vol. 63, no. 12, pp. 2521-2535, 2012.

[8] M. Rico, D. Camacho and Ó. Corcho, "A contribution-based framework for the creation of semantically-enabled web applications," Inf. Sci., vol. 180, no. 10, pp. 1850-1864, 2010.

[9] N. Noy and D. McGuinness, "Ontology development 101: a guide to creating your first ontology," 2001.

[10] J. Lee, et al, "Processing SPARQL queries with regular expressions in RDF databases," Bmc Bioinformatics, vol. 12, 2011.

[11] M. Grassi, "Developing HEO human emotions ontology," Biometric ID Management and Multimodal Communication, J. Fierrez, et al., Eds. Springer, vol. 5707, pp. 24-251, 2009.

[12] F.-M. Suchanek, F. Suchanek, G. Kasneci and G. Weikum, "YAGO: a large ontology from wikipedia and wordnet," Journal of Web Semantics, vol. 6 no.3, pp. 203-217, 2008. 